\title{
On Perturbations of the Periodic Toda Lattice
}

\author{
O. I. Bogoyavlensky
}

L. D. Landau Institute for Theoretical Physics,

The Academy of Sciences of the USSR, Moscow, USSR

\begin{abstract}
A class of Hamiltonian systems including perturbations of the periodic Toda lattice and homogeneous cosmological models is studied. Separatrix approximation of oscillation regimes in these systems connected with Coxeter groups is obtained. Hamiltonian systems connected with simple Lie algebras are pointed out, which generalize the system describing periodic Toda lattice and allow the $L-A$ pair representation.
\end{abstract}

\section{Introduction and Summary}

The Toda lattice is known [1-5] to be an infinite system of unit mass particles, whose interaction is determined by the potential

$$
V=\sum_{i} \exp \left(q_{i}-q_{i+1}\right),
$$

where $q_{i}$ is the displacement of the $i$-th particle from the equilibrium. The periodic Toda lattice is determined by $q_{i+n+1} \equiv q_{i}$ condition and has the Hamiltonian

$$
H=1 / 2 \sum_{i=1}^{n+1} p_{i}^{2}+\sum_{i=1}^{n} \exp \left(q_{i}-q_{i+1}\right)+\exp \left(q_{n+1}-q_{1}\right) .
$$

In the present paper we study the Hamiltonian systems generalizing (1.1):

$$
\begin{aligned}
\dot{p}_{i}=-\partial H / \partial q_{i}, \quad \dot{q}_{i}=\partial H / \partial p_{i} \\
\quad H=1 / 2 \sum_{i, j}^{n} a_{i j} p_{i} p_{j}+\sum_{k, m}^{n+1} b_{k m}\left(\left\{\alpha_{k}, q\right\}+\left\{\alpha_{m}, q\right\}\right) .
\end{aligned}
$$

Here $\alpha_{1}, \ldots, \alpha_{n+1}$ are vectors in $n$-dimensional space $R^{n}$ with coordinates $\alpha_{k}=$ $\left(d_{k 1}, \ldots, d_{k n}\right), q$ being the vector $\left(q_{1}, \ldots, q_{n}\right)$. In $R^{n}$ two scalar products $(x, y)$ and $\{x, y\}$ are given:

$$
(x, y)=\sum_{i, j}^{n} a_{i j} x_{i} y_{j}, \quad\{x, y\}=\sum_{i=1}^{n} x_{i} y_{i} .
$$


Vectors $\alpha_{k}$ and quadratic forms $a_{i j}, b_{k m}$ satisfy the conditions A and B:

A. For any vector $p$ in $R^{n}$

$\max _{k}\left(\alpha_{k}, p\right)>0$

B. For all $k$

$\left(\alpha_{k}, \alpha_{k}\right) b_{k k}>0$.

Among Hamiltonian systems (1.2) there are perturbations of the periodic Toda lattice (at the total zero momentum $p_{1}+\ldots+p_{n+1}=0$ ) with arbitrary masses of particles and periodic potential of the type, for instance:

$$
V_{1}=\sum_{i} \exp \left(q_{i}-q_{i+1}+\varepsilon_{i}\left(q_{i}-q_{i+2}\right)\right), \quad \varepsilon_{i+n+1} \equiv \varepsilon_{i} .
$$

The author has remarked, that Hamiltonian system (I.I) looks like Einstein dynamical systems in the theory of homogeneous cosmological models (hamiltonian of the most complicated model of type IX see (I.7) below). Qualitative theory of these models was constructed in the cycle of works by the author and Novikov [7-9]. The open set $S$ of physically permissible meanings of phase variables was marked out. The compactification of region $S$ was made and the boundary was "glued" then. On the initial stages of the Universe evolution the trajectories of Einstein dynamical system are near boundary $\Gamma$. The structure of the dynamical system on boundary $\Gamma$ turns out nontrivial: there are complicated sets of singular points and their separatrices. Close attractive subsystems, which are in fact so-called "attractors", may be marked out from these sets of separatrices. These attractors give birth to oscillation regimes with complicated ergodic properties (for the first time the regime of such type was discovered by Belinsky, Lifshits, Khalatnikov by other methods [15]). The results of qualitative theory were applied [7-10] for the study of the Universe expansion dynamics on the initial stages. Similar results for the hydrodynamical models were received in [10]. Note, that some hamiltonian systems of the type (1.2) on mapping

$$
Q_{k}=\exp \left(\left\{\alpha_{k}, g\right\}\right), \quad k=1, \ldots, n+1
$$

transform into systems of hydrodynamical type $\left(\right.$ at $\left.\alpha_{1}+\ldots+\alpha_{n+1}=0\right)$ in terms of paper [18].

Natural hypothesis has arisen that already in the perturbations of periodic Toda lattice of (1.2) type similar complicated oscillation regimes with ergodic properties spring up, and the Toda lattice is isolated by some deep algebraic symmetry.

Such regime at a positively determined metric $a_{i j}$ is realized at the energy $H \gg 1^{1}$. In this oscillatory regime momentum $p$ changes in the following way. There are long sections $\boldsymbol{I}_{N}$ of the trajectory along which momentum $p$ is approximately constant, momentum $p$ changes between two successive sections $\boldsymbol{I}_{N}$, $\boldsymbol{I}_{N+1}$ and

$$
p\left(\boldsymbol{I}_{N+1}\right)=T\left(p\left(\boldsymbol{I}_{N}\right)\right)=\tau_{k}\left(p\left(\boldsymbol{I}_{N}\right)\right)
$$

1 After this paper was completed Ya. G. Sinai acquainted the author with the new preprint [6] in which dynamics of the two particles system with arbitrary masses and Toda potential has been investigated numerically. The results [6] evidently point out the presence of stochastisity at $H \gg 1$. This agrees with the results of the given paper 
where number $k$ is determined by the condition

$$
\left(\alpha_{k}, p\left(\boldsymbol{I}_{N}\right)\right)=\max _{m}\left(\alpha_{m}, p\left(\boldsymbol{I}_{N}\right)\right)
$$

and map $\tau_{k}$ is the reflection in the plane orthogonal to vector $\alpha_{k}$ (in the metric $a_{i j}$ ):

$$
\tau_{k}(p)=p-\frac{2\left(\alpha_{k}, p\right)}{\left(\alpha_{k}, \alpha_{k}\right)} \alpha_{k} .
$$

The map $T$ given in (1.4)-(1.6) is almost everywhere determined in $R^{n}$ and is piecewise continuous.

The map $T$ for the Toda lattice (1.1) is periodic-here vectors $\alpha_{i}$ are the roots of the simple Lie algebra of type $A_{n}(\mathrm{SL}(n+1))$. The map $T$ will be periodic for the Hamiltonian systems (1.2), provided the Coxeter group $G$ generated by reflections (1.6) is finite (if $d$ is the order of group $G$, then $T^{d !} \cdot T^{d}=T^{d}$, the map $T$, however, may be irreversible). All the finite Coxeter groups $G$ are known [11], and, except for three special cases, they are Weyl groups of the simple Lie algebras (provided $G$ is not decomposed into a product of two other groups). The exceptions are two Coxeter groups in three- and four-dimensional spaces and the infinite series of dyadral groups (symmetry groups of regular polygons) in two-dimensional space.

The periodicity property of the map $T$ isolates the Toda lattice (1.1) among its general perturbations (1.2). In the general case (1.2) the Coxeter group $G$ is infinite and its closure (at positively determined metric $a_{i j}$ ) coincides with the orthogonal group $\boldsymbol{O}(\boldsymbol{n})$; for general vector $p$ the set $T^{k}(p)$ (where $k$ are all natural numbers) is everywhere dense on the sphere $(p, p)=$ const. The general Hamiltonian system (1.2) is unlikely to have any integrals, except for the energy $H$.

However, there are several exceptional systems of the (1.2) type with the periodic map $T$ and a large set of integrals. Hamiltonian systems of type (1.2) for each simple Lie algebra are presented in Section 4, allowing as well as the Toda lattice (1.1) [3-5] $L-A$ pair representation and having $n$ integrals. These systems and the way of their construction differ essentially from those investigated in papers [12-14].

Note, Hamiltonian systems of type (1.2) also arise in the theory of homogeneous cosmological models [15]. For instance, the model of type IX in empty space (at the level $H=0$ ) is described by the Hamiltonian system with Hamiltonian [7]

$$
H=2 \sum_{i<j}^{3} p_{i} p_{j}-\sum_{i=1}^{3} p_{i}^{2}+2 \sum_{i<j}^{3} \exp \left(q_{i}-q_{j}\right)-\sum_{i=1}^{3} \exp \left(2 q_{i}\right)
$$

Metric $a_{i j}$ for this case is indefinite and Coxeter group $G$ is infinite.

\section{Transformation of the Hamiltonian System}

The Hamiltonian system (1.2) by the mapping (1.3) is transformed into the system

$$
\begin{aligned}
\dot{p}_{i} & =-\sum_{k, m}^{n+1} b_{k m}\left(d_{k i}+d_{m i}\right) Q_{k} Q_{m}, \\
\dot{Q}_{k} & =Q_{k}\left(\sum_{i, j}^{n} d_{k i} a_{i j} p_{j}\right),
\end{aligned}
$$

determined on invariant submanifold $L$ :

$$
Q_{1}^{a_{1}} \cdot \ldots \cdot Q_{n+1}^{a_{n+1}}=1, \quad Q_{i}>0
$$


where

$$
a_{1} \alpha_{1}+\ldots+a_{n+1} \alpha_{n+1}=0 \text {. }
$$

Introduce new coordinates

$$
\begin{aligned}
& r_{k}=Q_{k} / G, \quad s_{i}=p_{i} / P, \quad w=G^{2} / P^{2}, \\
& G=\left(Q_{1}^{2}+\ldots+Q_{n+1}^{2}\right)^{1 / 2}, \quad P=\left(p_{1}^{2}+\ldots+p_{n}^{2}\right)^{1 / 2}
\end{aligned}
$$

and perform substitution of time

$$
d \tau_{1}=P d t
$$

System (2.1) in coordinates (2.2) and time $\tau_{1}$ has the form

$$
\begin{aligned}
\dot{r}_{k} & =r_{k}\left(\sum_{i, j} d_{k i} a_{i j} s_{j}-\sum_{m, i, j} r_{m}^{2}\left(d_{m i} a_{i j} s_{j}\right)\right), \\
\dot{s}_{i} & =w\left(-\sum_{k, m} b_{k m}\left(d_{k i}+d_{m i}\right) r_{k} r_{m}+s_{i} \sum_{k, j, m} s_{j} b_{k m}\left(d_{k j}+d_{m j}\right) r_{k} r_{m}\right), \\
\dot{w} & =2 w\left(\sum_{i, j, k} r_{k}^{2}\left(d_{k i} a_{i j} s_{j}\right)+w \sum_{i, k, m} s_{i} b_{k m}\left(d_{k i}+d_{m i}\right) r_{k} r_{m}\right) .
\end{aligned}
$$

As a result of transformations of the coordinates (1.3), (2.2), the system (1.2) (manifold $L$ ) transforms into the system (2.3) determined in the region $S_{1}$

$$
w>0, \quad r_{k}>0
$$

on the $2 n$-dimensional invariant manifold $V$ :

$$
r_{1}^{2}+\ldots+r_{n+1}^{2}=1, \quad s_{1}^{2}+\ldots+s_{n}^{2}=1, \quad w \geqq 0 .
$$

System (2.3) is sure to continue on the closure $S$ of region $S_{1}$, where

$$
w \geqq 0, \quad r_{k} \geqq 0 .
$$

The components of the boundary $\Gamma$ of manifold $S\left(w=0, r_{k}=0\right)$ are invariant submanifolds of system (2.3).

\section{Separatrix Approximation of the Oscillatory Regime}

System (2.3) has $n+1$ sets $M_{i}(i=1, \ldots, n+1)$ of singular points. Each set $M_{i}$ is an $(n-1)$-dimensional sphere and has coordinates

$$
r_{k}=\delta_{k i}, \quad w=0, \quad s_{1}^{2}+\ldots+s_{n}^{2}=1 .
$$

The point $s$ on the set $M_{k}$ is denoted by a pair of $\{s, k\}$.

The eigenvalues of system (2.3) on the manifold $V$ in the singular point $\{s, k\}$ are the following (the corresponding eigen directions are shown in brackets):

$$
\begin{aligned}
\lambda_{m} & =\left(\alpha_{m}, s\right)-\left(\alpha_{k}, s\right) & & \text { (variable } \left.r_{m}, m=1, \ldots, n+1, m \neq k\right) \\
\lambda_{n} & =2\left(\alpha_{k}, s\right) & & (\text { variable } w) \\
\lambda_{n+1} & =\ldots=\lambda_{2 n}=0 & & \left(\text { variables } s_{i}\right) .
\end{aligned}
$$

It follows from condition A (Section 1) and (3.1) that all singular points $M_{k}$, except for submanifolds of less dimension, are nondegenerated and unstable 
(the singular point is called nondegenerated, provided the number of its zero eigenvalues is equal to the dimension of the set $M_{k}$ ).

Define two subsets $V_{k}$ and $W_{k}$ on each $M_{k}$ :

$$
\begin{array}{lll}
\text { on } \quad V_{k} & \left(\alpha_{k}, s\right)<0 \\
\text { on } \quad W_{k} & \left(\alpha_{k}, s\right)=\max _{m}\left(\alpha_{m}, s\right)>0 .
\end{array}
$$

Separatrices going from the point $\left\{s^{\circ}, j\right\}$ (at $\tau_{1} \rightarrow-\infty$ ) on the set $V_{j}$ pass (at $\left.\tau_{1} \rightarrow+\infty\right)$ to the point $\left\{s^{\circ}, k\right\}$ on the set $W_{k}$ (almost all) and have the form

$$
\begin{aligned}
& r_{m}\left(\tau_{1}\right)=c_{m} \exp \left(\left(\alpha_{m}, s^{\circ}\right) \tau_{1}\right)\left(\sum_{l} c_{l}^{2} \exp \left(2\left(\alpha_{l}, s^{\circ}\right) \tau_{1}\right)\right)^{-1 / 2}, \\
& w\left(\tau_{1}\right) \equiv 0, \quad s_{i}\left(\tau_{1}\right) \equiv s_{i}^{0}, \quad c_{m} \geqq 0 .
\end{aligned}
$$

Here $m, l$ are numbers $i$, for which $\lambda_{i}>0$ [see (3.1)]; in case $\lambda_{i}<0 r_{i} \equiv 0$.

The separatrix going from the point $\left\{s^{\circ}, k\right\}$ on the set $W_{k}$ passes to the point $\left\{\theta \tau_{k}\left(s^{\circ}\right), k\right\}$ on the set $V_{k}$, where $\tau_{k}$ is the reflection (1.6) and $\theta$ is the projection on the sphere $M_{k}$ (by rays passing through the centre). This separatrix in the coordinates $p_{i}, w$ and time $\tau$ :

$$
d \tau=2 w\left|b_{k k}\right| P^{2} d t
$$

has the form

$$
\begin{aligned}
p_{i}(\tau) & =p_{i}^{\circ}-\tau \sigma \alpha_{k}, & w(\tau) & =\frac{\left(\alpha_{k}, p^{\circ}\right) \tau-\sigma / 2\left(\alpha_{k}, \alpha_{k}\right) \tau^{2}}{\left\{p^{\circ}-\tau \alpha_{k}, p^{\circ}-\tau \alpha_{k}\right\}}, \\
r_{i}(\tau) & \equiv \delta_{i k}, & \sigma & =\operatorname{sign} b_{k k} .
\end{aligned}
$$

Note, system (1.3) after transformation into coordinates $p_{i}, r_{i}, w$ has the $n$ dimensional singular sets $R_{k}^{n}$, corresponding to $M_{k}$ (on $R_{k}^{n}$ momenta $p_{i}$ are arbitrary). The separatrices of singular points $R_{k}^{n}$ in the coordinates $s_{i}, r_{i}, w$ turn into those of singular points $M_{k}$.

The separatrix (3.3) at $\tau=0$ goes from the singular point $p^{\circ}$. At

$$
\tau=2 \sigma\left(\alpha_{k}, p^{\circ}\right) /\left(\alpha_{k}, \alpha_{k}\right)>0
$$

[here condition B (Section 1) is used] separatrix (3.3) is inserted into the singular point

$$
p^{1}=\tau_{k}\left(p^{\circ}\right)=p^{\circ}-\frac{2\left(\alpha_{k}, p^{\circ}\right)}{\left(\alpha_{k}, \alpha_{k}\right)} \alpha_{k}, \quad w=0,
$$

belonging to the set $V_{k}$, since

$$
\left(\alpha_{k}, p^{1}\right)=-\left(\alpha_{k}, p^{\circ}\right)<0 \text {. }
$$

Thus, the map $T_{1}$ is determined on sets $V_{j}, W_{k}$

$$
T_{1}: V_{j} \rightarrow W_{k}, \quad T_{1}: W_{k} \rightarrow V_{k}, \quad(j, k=1, \ldots n+1),
$$

where the arrow denotes separatrix [(3.2) or (3.3)] going from the initial point to the finite one. The trajectory of the system (2.3) initiating in the neighbourhood of one of the sets $V_{j}, W_{k}$ will move along the succession of separatrices

$$
\ldots \rightarrow V_{j} \rightarrow W_{k} \rightarrow V_{k} \rightarrow W_{i} \rightarrow \ldots \text {. }
$$


Due to (3.2) or (3.3) the corresponding trajectory in the initial coordinates $q_{i}$ moves in the first approximation as follows: at the transition along separatrix (3.2) the motion along straight line proceeds with the constant momentum $p_{i}$, this motion terminates when some $Q_{k} \gg Q_{m}$ for all $m$. Then at the transition along separatrix (3.3) an effective "reflection" of the momentum $p_{i}$ described by the map $\tau_{k}(1.6)$ occurs. Then the motion along a straight line with the constant momentum $p_{i}^{1}=\tau_{k}\left(p_{i}\right)$ follows again, etc.

This oscillatory regime is realized at $H \gg 1$ in the case of a positively determined metrix $a_{i j}$ [see (1.2)]. Indeed, from condition A (Section 1) follows

$$
w=G^{2} / P^{2} \geqq 1 / P^{2} \text {. }
$$

The energy $H$ in the coordinates (2.2) has the form

$$
H=1 / 2 P^{2}\left(\sum_{i, j} a_{i j} s_{i} s_{j}+w \sum_{k, m} b_{k m} r_{k} r_{m}\right) .
$$

Since at the transition along separatrix (3.2) $w \ll 1$, at the positively determined metric $a_{i j}$ we get $H \gg 1$. For the case of the indefinite metric $a_{i j}$, as (1.7) shows, the oscillatory regime is also realized at $H=0$. (See discussion in Section 1).

\section{The Hamiltonian Systems Connected with Simple Lie Algebras}

This Section presents new examples of Hamiltonian systems of the (1.2) type, which, as well as the Toda lattice (1.1), assume a representation in the $L-A$ pair form. The theory of simple Lie algebras $\mathbb{5}$ is used for constructing these systems.

Summarize the data on the Cartan-Weyl basis $e_{\alpha_{i}}, h_{k}$ in $(\mathfrak{5}$ [11], necessary for the subsequent analysis. In the Cartan subalgebra $H$ ( $H$ - the maximum commutative subalgebra in (5) there is a set of vectors $\alpha_{1}, \ldots, \alpha_{S}$ called roots, and some basis $h_{1}, \ldots, h_{n}$ is chosen $(n=\operatorname{dim} H$ is called a rank of algebra (5). Vectors $e_{\alpha_{i}}, h_{k}$ form the basis of the algebra $(\mathfrak{5}$ and satisfy the commutation relations:

$$
\begin{array}{ll}
{\left[e_{\alpha_{i}}, e_{\alpha_{j}}\right]=N_{\alpha_{i} \alpha_{j}} e_{\alpha_{i}+\alpha_{j}},} & {\left[e_{\alpha_{i}}, e_{-\alpha_{i}}\right]=\alpha_{i},} \\
{\left[h_{k}, e_{\alpha_{i}}\right]=\left(h_{k}, \alpha_{i}\right) e_{\alpha_{i}},} & {\left[h_{i}, h_{j}\right]=0 .}
\end{array}
$$

The scalar product $(x, y)$ is determined by the Killing-Cartan form

$$
(x, y)=\operatorname{Tr}(\operatorname{ad} x \circ \operatorname{ad} y), \quad \operatorname{ad} x(z)=[x, z] .
$$

The set of roots $\alpha_{1}, \ldots, \alpha_{N}$ is named admissible, provided vectors $\alpha_{i}-\alpha_{j}$ for all $i, j \leqq N$ are not a root; hence

$$
\left[e_{\alpha_{i}}, e_{-\alpha_{j}}\right]=0 \text {. }
$$

In each simple Lie algebra $\mathfrak{5}$ there is one important admissible set of roots

$$
\omega_{1}, \omega_{2}, \ldots, \omega_{n},-\Omega \text {, }
$$

where $\omega_{i}$ are simple roots (all the roots $\alpha_{i}$ are integer linear combinations of $\left.\omega_{k}\right)$ and $\Omega$ is the so-called maximum root $\left(\Omega+\ell_{1} \omega_{1}+\ldots+\ell_{n} \omega_{n}\right.$ is not the root at 
$\ell_{i} \geqq 0$ ). All subsets of an admissible set of roots are also admissible. Prove the following

Theorem 1. Let $0 \mathfrak{b}$ be a simple Lie algebra of rank $n, h_{1}, \ldots, h_{n}-$-a basis of its Cartan subalgebra, $\alpha_{1}, \ldots, \alpha_{N}$-an admissible set of roots, $\alpha_{i}=d_{i 1} h_{1}+\ldots+d_{i n} h_{n}, b_{j}$-arbitrary real constants, the scalar product $(x, y)$ in $\mathbf{6}$ is determined by the Killing-Cartan form. The Hamiltonian system in the $2 n$-dimensional phase space $p_{i}, q_{i}$ :

$$
\begin{aligned}
& \dot{p}_{i}=-\partial H / \partial q_{i}, \quad \dot{q}_{i}=\partial H / \partial p_{i}, \\
& H=1 / 2 \sum_{k, m}^{n}\left(h_{k}, h_{m}\right) p_{k} p_{m}+\sum_{j=1}^{N} b_{j} \exp \left(2 \sum_{k=1}^{n} d_{j k} q_{k}\right)
\end{aligned}
$$

is asserted to have a representation in the $L-A$ pair form.

Proof. All the solutions of system (4.3) by the map

$$
l_{j}=b_{j}^{1 / 2} \exp \left(\sum_{k=1}^{n} d_{j k} q_{k}\right), \quad j=1, \ldots, N
$$

are transformed into the solution of system

$$
\begin{aligned}
\dot{p}_{k} & =-2 \sum_{j=1}^{N} l_{j}^{2} d_{j k}, \\
\dot{l}_{j} & =l_{j} \sum_{k, m}^{n} d_{j m} p_{k}\left(h_{k}, h_{m}\right) .
\end{aligned}
$$

Consider in the Lie algebra 65 the equation [17]

$$
i=[l, A(l)]
$$

where vectors $l(t)$ and $A(l(t))$ have the form

$$
\begin{aligned}
l(t) & =\sum_{j=1}^{N} l_{j}(t)\left(e_{\alpha_{j}}+e_{-\alpha_{j}}\right)+\sum_{k=1}^{n} p_{k} h_{k}, \\
A(l(t)) & =\sum_{j=1}^{N} l_{j}(t)\left(e_{\alpha_{j}}-e_{-\alpha_{j}}\right) .
\end{aligned}
$$

Using the commutation relations (4.1) and the determination of the admissible set of roots $\alpha_{1}, \ldots, \alpha_{N}$, it is easy to verify that system (4.5) is equivalent to Equation (4.6) [under condition (4.7)].

Equation (4.6) at any linear representation $T$ of the Lie algebra $\mathbb{5}$ determines the $L-A$ pair

$$
T(l)^{\circ}=[T(l), T(A(l))] .
$$

Thus, by mappings (4.4), (4.7) all the solutions of system (4.3) transfer to solutions of Equation (4.6) and, consequently, equation (4.8). Take an exact representation $T$ of algebra $(5$ with minimal dimension. Then Equation $(4.8)$ at $l(t)$ given by $(4.7)$ and (4.4) will be equivalent to system (4.3). This proves Theorem 1.

It follows [16] from the representation in the $L-\mathrm{A}$ pair form that system (4.3) has integrals

$$
\boldsymbol{I}_{k}=\operatorname{Tr}\left(T^{k}(l(t))\right) \text {. }
$$

Equation (4.6) always has integral $(l, l)\left(\boldsymbol{I}_{2}\right)$ which under condition (4.7) has the form

$$
(l, l)=\sum_{k, m}^{n}\left(h_{k}, h_{m}\right) p_{k} p_{m}+2 \sum_{j=1}^{N} l_{j}^{2}=2 H .
$$


Here are some examples of systems (4.3). We use the classification of simple Lie algebras and standard list of algebra $6 \mathfrak{5}$ roots in orthogonal basis $e_{1}, \ldots, e_{n}$ (see [11]) (for the algebras of types $A_{n}, E_{6}, E_{7}, G_{2}$ it is convenient to expand the Cartan subalgebras by the element commutating with the total algebra; in this expansion we have the basis $\left.e_{1}, \ldots, e_{n}, e_{n+1}\right)$. The orthogonal basis $e_{i}$ is taken as basis $h_{i}$, and set (4.2) is taken as admissible set of roots. The corresponding Hamiltonian (4.3) has the form:

$$
H=1 / 2 \sum_{i=1}^{m} p_{i}^{2}+V_{\mathfrak{F}}\left(q_{i}\right)
$$

Here $m=n+1$ for the algebras of types $A_{n}, E_{6}, E_{7}, G_{2}$ and $m=n$ for: the rest of the types ( $n$ is a rank of algebra $(\mathfrak{5})$.

Denote

$$
V_{k}=\sum_{i=1}^{k} \exp \left(q_{i}-q_{i+1}\right) .
$$

The explicit form of potentials $V_{\mathfrak{G}}\left(q_{i}\right)$ depending on the type of algebra 65 is the following:

$$
\begin{aligned}
V_{A_{n}}= & V_{n}+\exp \left(q_{n+1}-q_{1}\right), \quad n \geqq 2, \\
V_{B_{n}}= & V_{n-1}+\exp \left(q_{n}\right)+\exp \left(-q_{1}-q_{2}\right), \quad n \geqq 2, \\
V_{C_{n}}= & V_{n-1}+\exp \left(2 q_{n}\right)+\exp \left(-2 q_{n}\right), \quad n \geqq 3, \\
V_{D_{n}}= & V_{n-1}+\exp \left(q_{n-1}+q_{n}\right)+\exp \left(-q_{1}-q_{2}\right), \quad n \geqq 4, \\
V_{E_{6}}= & V_{5}+\exp \left(1 / 2\left(-q_{1}-q_{2}-q_{3}+q_{4}+q_{5}+q_{6}\right)+q_{7} / \sqrt{2}\right)+\exp \left(-\sqrt{2} q_{7}\right) \\
V_{E_{7}}= & V_{5}+\exp \left(1 / 2\left(-q_{1}+q_{2}+\ldots+q_{7}-q_{8}\right)\right)+\exp \left(-q_{1}-q_{2}\right)+\exp \left(-q_{7}+q_{8}\right), \\
V_{E_{8}}= & V_{6}+\exp \left(1 / 2\left(-q_{1}+q_{2}+\ldots+q_{7}-q_{8}\right)\right)+\exp \left(-q_{1}-q_{2}\right)+\exp \left(q_{7}+q_{8}\right), \\
V_{F_{4}}= & \exp \left(q_{1}-q_{2}\right)+\exp \left(q_{2}-q_{3}\right)+\exp \left(q_{3}\right)+\exp \left(1 / 2\left(-q_{1}-q_{2}-q_{3}+q_{4}\right)\right) \\
& +\exp \left(-q_{1}-q_{4}\right), \\
V_{G_{2}}= & \exp \left(q_{1}-q_{2}\right)+\exp \left(-2 q_{1}+q_{2}+q_{3}\right)+\exp \left(q_{1}+q_{2}-2 q_{3}\right) .
\end{aligned}
$$

Using standard linear representations of simple Lie algebras, the Hamiltonians (4.9) may be shown to have $m$ integrals; for some cases it is possible to prove that integrals are in involution.

For Lie algebra of type $A_{n}(\mathrm{SL}(n+1))$ the Hamiltonian (4.9) determines the periodic Toda lattice, for the rest of the types we obtain new systems of particles with a large number of integrals [note the system (4.9) for the type $C_{n}$ is inserted into the periodic Toda lattice with $2 n$ particles]. Stochasticity is impossible in all these systems.

For the two-particle systems from (4.10) in addition to the Toda lattice we get two integrable Hamiltonian systems with potentials

$$
\begin{aligned}
& V_{B_{2}}=\exp \left(q_{1}-q_{2}\right)+\exp \left(q_{2}\right)+\exp \left(-q_{1}-q_{2}\right), \\
& V_{G_{2}}=\exp \left(q_{1}\right)+\exp \left(\sqrt{3} q_{2}\right)+\exp \left(-3 / 2 q_{1}-\sqrt{3} / 2 q_{2}\right) .
\end{aligned}
$$


Note, that both these systems differ from that considered in [6] with the potential

$$
V_{T}=\exp \left(q_{1}-q_{2}\right)+\exp \left(q_{2}\right)+\exp \left(-q_{1}\right)
$$

describing the Toda lattice with one fixed particle.

Basing on Theorem 1 we may indicate many other examples of Hamiltonian systems admitting representation in the $L-A$ pair form, for instance, such systems result from (4.9) by omitting some terms in potential $V_{\mathfrak{G}}$. One of such systems (for type $A_{n}$ ) has been recently studied in detail by J.Moser.

\section{References}

1. Toda, M.: Progr. Theor. Phys. Suppl. 45, 174 (1970)

2. Henon, M.: Phys. Rev. B 9, 1921 (1974)

3. Flascka, H.: Phys. Rev. B 9, 1924-1925 (1974)

4. Manakov, S. V.: Soviet Zhurn. Eksp. Teor. Fiz. 67, 543-555 (1974)

5. Dubrovin, B.A., Matveev, V.B., Novikov, S.P.: Soviet Usp. Math. Science 31, $56-136$ (1976)

6. Cassati, G., Ford, J.: Phys. Rev. A 12, 1702-1709 (1975)

7. Bogoyavlensky, O.I., Novikov, S.P.: Soviet Zhurn. Eksp. Teor. Fiz. 64, 1475-1495 (1973)

8. Bogoyavlensky, O. I., Novikov, S. P.: Proceedings of the Seminar by I. G. Petrovsky I, 7 -42 (1975)

9. Bogoyavlensky, O. I.: Soviet Zhurn. Eksp. Teor. Fiz. 70, 361-373 (1976)

10. Bogoyavlensky, O. I.: Soviet Appl. Math. Mech. 40, 2, 270-280 (1976)

11. Bourbaki, N.: Groupes et algebras de Lie. Paris: Hermann 1968

12. Moser, J.: Advan. Math. 16, 197-220 (1975)

13. Calogero,F., Ragnisco, O., Matchioro, C.: Lett. Nuovo Cimento 13, 383-388 (1975)

14. Olshanetsky, M.A., Ferelomov, A. M.: To be published in Lett. Math. Phys.

15. Landau, L.D., Lifshitz,E. M.: Classical Theory of Fields. Moscow: Nauka 1973

16. Lax,P.D.: Commun. Pure Appl. Math. 21, 467-491 (1968)

17. Arnold, V.I : Ann. Inst. Fourier (Grenoble) 16, 319-361 (1966)

18. Obouknov, A. M.: Fluid Dynam. Trans. 5, 193-200 (1971)

Communicated by J. Moser

Received June 22, 1976 
